ARTICLE

Received 31 Mar 2014 | Accepted 8 Jul 2014 | Published 21 Aug 2014 DOI: 10.1038/ncomms5638

\title{
Ubiquitin-binding site 2 of ataxin-3 prevents its proteasomal degradation by interacting with Rad23
}

\author{
Jessica R. Blount ${ }^{1,2, \star}$, Wei-Ling Tsou ${ }^{1,2, \star}$, Gorica Ristic ${ }^{1}$, Aaron A. Burr ${ }^{1,3}$, Michelle Ouyang ${ }^{1}$, Holland Galante ${ }^{4}$, \\ K. Matthew Scaglione ${ }^{4} \&$ Sokol V. Todi ${ }^{1,2,3}$
}

Polyglutamine repeat expansion in ataxin-3 causes neurodegeneration in the most common dominant ataxia, spinocerebellar ataxia type 3 (SCA3). Since reducing levels of disease proteins improves pathology in animals, we investigated how ataxin-3 is degraded. Here we show that, unlike most proteins, ataxin-3 turnover does not require its ubiquitination, but is regulated by ubiquitin-binding site 2 (UbS2) on its $\mathrm{N}$ terminus. Mutating UbS2 decreases ataxin-3 protein levels in cultured mammalian cells and in Drosophila melanogaster by increasing its proteasomal turnover. Ataxin-3 interacts with the proteasome-associated proteins Rad23A/B through UbS2. Knockdown of Rad23 in cultured cells and in Drosophila results in lower levels of ataxin-3 protein. Importantly, reducing Rad23 suppresses ataxin-3dependent degeneration in flies. We present a mechanism for ubiquitination-independent degradation that is impeded by protein interactions with proteasome-associated factors. We conclude that UbS2 is a potential target through which to enhance ataxin-3 degradation for SCA3 therapy.

\footnotetext{
${ }^{1}$ Department of Pharmacology, Wayne State University School of Medicine, 540 E Canfield, Scott Hall Room 3108, Detroit, Michigan 48201, USA. ${ }^{2}$ Department of Neurology, Wayne State University School of Medicine, 540 E Canfield, Scott Hall Room 3108, Detroit, Michigan 48201, USA. ${ }^{3}$ Cancer Biology Program, Wayne State University School of Medicine, 540 E Canfield, Scott Hall Room 3108, Detroit, Michigan 48201, USA. ${ }^{4}$ Department of Biochemistry and Neuroscience Research Center, Medical College of Wisconsin, BC038, 8701 Watertown Plank Road, Milwaukee, Wisconsin 53226, USA

* These authors contributed equally to this work. Correspondence and requests for materials should be addressed to S.V.T. (email: stodi@med.wayne.edu).
} 
S pinocerebellar ataxia type 3 (SCA3), which is also known as Machado-Joseph disease, is an age-related neurodegenerative disease that belongs to the family of polyglutamine (polyQ)-dependent disorders ${ }^{1,2}$. The maladies that comprise this group result from abnormal expansions in the polyQ region of different proteins. PolyQ diseases also include Huntington's, spinobulbar muscular atrophy, dentatorubral-pallidoluysian atrophy and five more $\mathrm{SCAs}^{2-4}$.

SCA3, considered to be the most common dominantly inherited ataxia in the world, is caused by an abnormal CAG expansion in the ATXN3 gene that is normally 12-42 repeats in length, but is expanded to $\sim 52-84$ repeats in diseased individuals ${ }^{1}$. PolyQ expansion occurs in the protein ataxin-3, a deubiquitinase (DUB) that is involved in protein quality control. Ataxin-3 protects mammalian cells against various forms of stress $^{5}$ and serves a protective role against toxic polyQ proteins in Drosophila $^{6,7}$. How pathogenic expansion of the polyQ region of ataxin-3 causes SCA3 is unknown. There is presently no cure for this disease.

Reducing the levels of disease proteins improves degeneration in various animal models of proteinopathies, including polyQ diseases ${ }^{3,8-17}$. Consequently, a potential therapeutic strategy for SCA3 entails reducing the levels of the ataxin-3 protein. This concept is supported by recent work that used viral-mediated delivery of RNA-interference (RNAi) constructs to reduce levels of ataxin- 3 protein in mouse brain ${ }^{18}$, and alleviated SCA3-like pathology in one study ${ }^{19}$.

The mechanism of degradation of the ataxin-3 protein is not clear $^{1}$. Most cellular proteins are degraded by being posttranslationally modified with the small protein ubiquitin, which targets them for proteasomal degradation ${ }^{20}$. Here, we present evidence that ubiquitination of ataxin-3 is not necessary for its proteasomal degradation, and find that the turnover of this polyQ protein is regulated by the ubiquitin-binding site 2 (UbS2) on its $\mathrm{N}$ terminus. UbS2 mediates the interaction of ataxin-3 with the proteasome-associated proteins $\operatorname{Rad} 23 \mathrm{~A}$ and $\operatorname{Rad} 23 \mathrm{~B}^{21-24}$. According to our studies, this interaction prevents the proteasomal turnover of ataxin-3. Our findings describe a precise molecular target through which to enhance the degradation of ataxin-3 protein for SCA3 therapeutics, perhaps by designing molecules that bind UbS2 and prevent its interaction with $\operatorname{Rad} 23 \mathrm{~A} / \mathrm{B}$.

\section{Results}

Ataxin-3 does not require ubiquitination to be degraded. Previous reports presented evidence that the turnover of normal and pathogenic ataxin-3 (Fig. 1a) depends on the proteasome ${ }^{1}$, and we showed that ataxin-3 protein is ubiquitinated in mammalian cells, in mouse brain and in Drosophila ${ }^{7,25-29}$. On the basis of these observations, one would expect ataxin- 3 to be degraded by becoming ubiquitinated and thus targeted for the proteasome. Yet, while the role of the proteasome in ataxin-3 degradation is well supported ${ }^{1}$, it is not entirely clear that ataxin-3 needs to be ubiquitinated to be degraded.

To examine this possibility, we used a version of ataxin-3 whose lysine residues are mutated into the similar but nonubiquitinatable amino-acid arginine (K-null). We do not detect ubiquitinated species with K-null ataxin-3 in cultured mammalian cells (Fig. 1b) or in Drosophila (Fig. 2b, as well as other supportive data published by us ${ }^{7,27}$ ). Mutating all of the 15 lysine residues of ataxin-3 into arginines does not affect its subcellular localization in mammalian cells, or disrupt its ability to cleave ubiquitin chains in reconstituted systems in vitro ${ }^{27}$. Lysine-less ataxin-3 retains some of its functions: similarly to the wild-type (WT) version of this DUB, the K-null variant is able to somewhat suppress neurodegeneration in Drosophila, although this function cannot be enhanced by its own ubiquitination to reach the full protective effect of ubiquitinatable ataxin-3 (ref. 7).

We first examined the turnover of K-null ataxin-3 protein compared with its wild-type counterpart in cultured cells. Figure 1c summarizes cycloheximide (CHX)-based pulse-chase studies, where we observe that the turnover of K-null ataxin-3 is not slower than that of WT ataxin-3. In fact, based on quantification of data from multiple western blots, K-null ataxin-3 might be degraded slightly more quickly than the normal version of this DUB in cells (Fig. 1c, 360 min time point; Fig. 1d, $120 \mathrm{~min}$ time point). Both WT and K-null ataxin-3 proteins are stabilized in cells by the addition of the proteasome inhibitor MG132 (Fig. 1d), a finding that is in accordance with previous reports that ataxin-3 turnover in cells is proteasome dependent ${ }^{1,25}$.

Subsequently, we used the fruit fly Drosophila melanogaster to investigate whether K-null ataxin-3 accumulates in vivo in an intact organism. We utilized the Gal4-UAS system ${ }^{30}$ to express ataxin- 3 constructs throughout the $\mathrm{fly}^{7}$. We generated flies that express WT, K-null or K-117 (the lysine predominantly ubiquitinated in vitro and in mammalian cell culture ${ }^{27}$ ) ataxin-3 by using the ubiquitous Gal4 driver sqh-Gal4 (refs 31,32 ). We compared fly lines that express the various ataxin-3 transgenes at similar messenger RNA (mRNA) levels, according to quantitative reverse transcriptase-PCR (Fig. 2a). As shown in Fig. 2b, K-null ataxin-3 protein does not accumulate compared with WT or K-117 ataxin-3. Together, results in Figs 1 and 2 lead us to conclude that ubiquitination of ataxin-3 is not absolutely necessary for its degradation.

UbS2 regulates ataxin-3 protein levels and turnover in cells. Since ubiquitination of ataxin-3 does not appear to be necessary for its turnover (Figs 1 and 2), we wondered whether proteinbinding domains of this DUB are involved in its degradation. Several domains on ataxin-3 enable its direct interaction with other proteins (Fig. 1a). Starting at the $\mathrm{N}$ terminus, first is UbS1 on the catalytic domain. UbS1 binds ubiquitin and is necessary for the catalytic activity of ataxin-3 (ref. 23). Next is UbS2, which binds ubiquitin, Rad23A and Rad23B (refs 21,23). Rad23A/B facilitate substrate protein degradation by reversibly associating with the proteasome ${ }^{33,34}$. Previous structural work focusing on the catalytic domain of ataxin-3 indicated that mutating the amino-acid tryptophan (W) at position 87 disrupts the ability of UbS2 to interact with the isolated ubiquitin-like domain of Rad23 (refs 21,23). Through an in vitro pull-down assay that utilized recombinant, full-length ataxin-3 and Rad23A, we confirmed that mutating W87 into an alanine residue diminishes the ability of these two proteins to interact (Supplementary Fig. 1a). We also verified in mammalian cell culture that this mutation reduces the capacity of an otherwise normal, full-length ataxin-3 to co-immunoprecipitate endogenous $\operatorname{Rad} 23 \mathrm{~A}$ and $\operatorname{Rad} 23 \mathrm{~B}$ (Supplementary Fig. 1b). Mutating UbS2 does not abrogate the catalytic activity of ataxin-3 (ref. 23) or affect its subcellular localization (Supplementary Fig. 2). In the C-terminal half of ataxin-3 are three ubiquitin-interacting motifs (UIMs), which bind ubiquitin chains at least four moieties long 29 . The C-terminal portion also contains an arginine-rich region that binds the AAA ATPase protein valosin-containing protein (VCP) (also known as p97) ${ }^{35-37}$. VCP functions at least in part as a proteasomal shuttle protein ${ }^{33}$. Mutating one or more of the arginine residues of the VCP-binding site of full-length ataxin-3 into histidines or alanines has been demonstrated to disrupt its interaction with VCP in reconstituted systems in vitro and in mammalian cells ${ }^{35-37}$. 

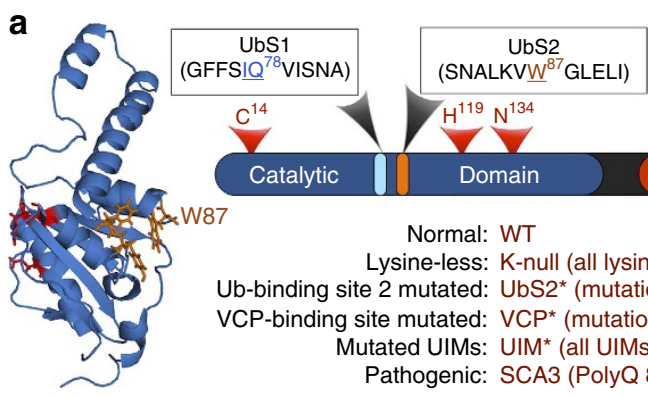

Lysine-less: K-null (all lysines mutated $\mathrm{K} \rightarrow \mathrm{R}$ ) Ub-binding site 2 mutated: UbS2* (mutation W87 $\rightarrow$ A or W87 $\rightarrow \mathrm{K}$ ) VCP-binding site mutated: VCP* (mutation RKRR $\rightarrow$ AAAA)

Mutated UIMs: UIM* (all UIMs mutated; $\mathrm{S} \rightarrow \mathrm{A}$ )

Pathogenic: SCA3 (PolyQ 81-84)
C

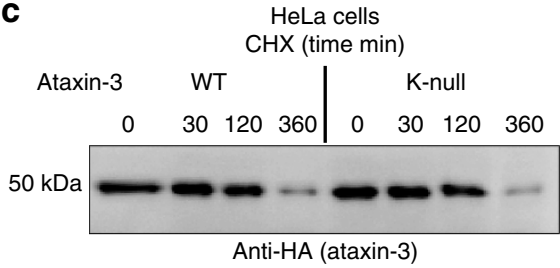

$50 \mathrm{kDa}$
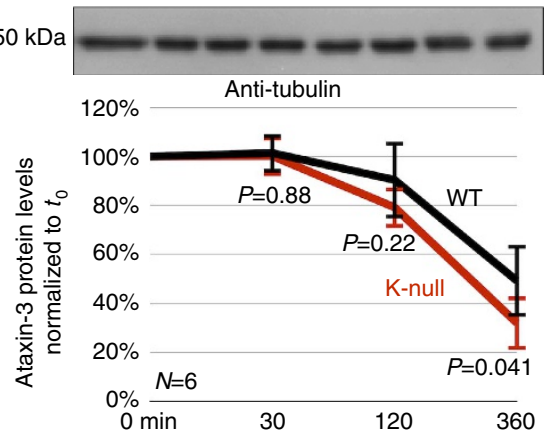

VCP-binding site (SEELRKRR ${ }^{285}$ EAYFEKQQQ)

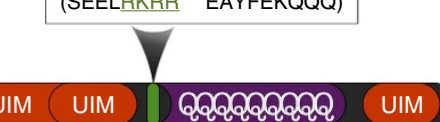

b

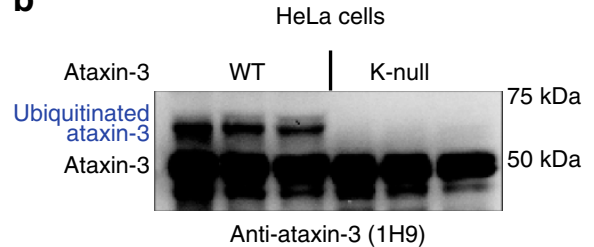

d $\begin{gathered}\text { HeLa cells } \\ \mathrm{CHX}(\text { time min) }\end{gathered}$

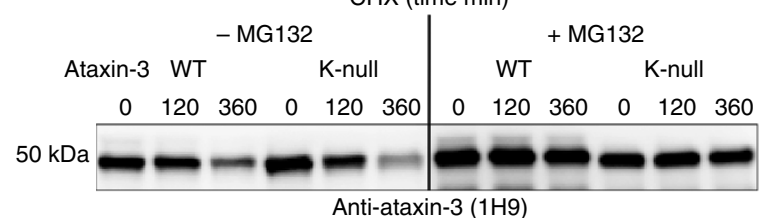

$50 \mathrm{kDa}$

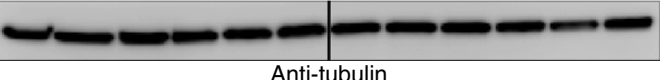

Anti-tubulin

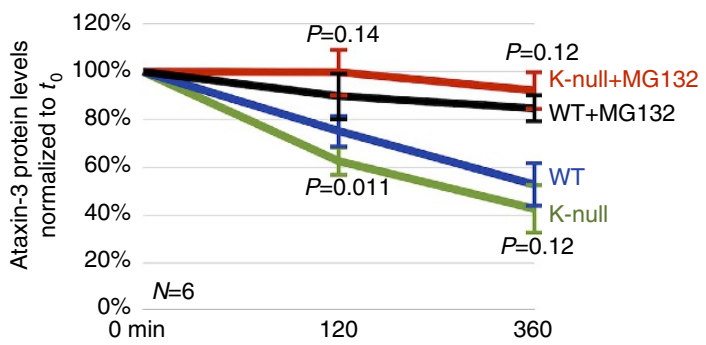

Figure 1 | Cellular degradation of ataxin-3 does not require its own ubiquitination. (a) Schematic of ataxin-3. The catalytic triad (red) and two ubiquitinbinding sites (UbS1, light blue and UbS2, orange) are on the catalytic domain (blue). Next are three ubiquitin-interacting motifs (UIMs; red), separated by the polyglutamine (polyQ) region. Preceding the polyQ is the VCP-binding site (green). The NMR-based structure of the catalytic domain of ataxin-3 was reported in ref. 22. The file for the structure depicted here was obtained from NCBI (PDB: 1YZB) and was rendered and annotated using the software application MacPyMOL. The legend included in panel a summarizes the abbreviations used throughout the text for the different ataxin-3 mutations. (b) Western blot of whole-cell lysates. HeLa cells were transfected with the indicated constructs and treated with MG132 (6h, $15 \mu \mathrm{M}) 24 \mathrm{~h}$ post transfection to enhance the capture of ataxin-3 ubiquitination, as we have described before ${ }^{26,27}$. We previously showed through stringent immunopurification protocols and mass spectrometry that the more slowly migrating band of ataxin-3 is ubiquitinated ataxin-325-28,55. Equal total protein loaded (50 $\mu \mathrm{g}$ per lane). Transfections were performed independently in triplicate. Overexposed blot to highlight ubiquitinated ataxin-3. (c) Top: western blots of whole-cell lysates. HeLa cells were transfected as indicated and treated for the specified amounts of time with cycloheximide (CHX) $48 \mathrm{~h}$ post transfection. Bottom: means of ataxin-3 signal quantified from blots on the top and other similar experiments. $P$-values are from Student's $t$-tests comparing protein levels of K-null ataxin-3 to the wild-type (WT) counterpart. Error bars: s.d. $N=6$ independently conducted experiments. (d) Top: western blots of whole-cell lysates of HeLa cells transfected as indicated, treated or not for $2 \mathrm{~h}$ with the proteasome inhibitor MG132 (10 $\mu \mathrm{M}) 24 \mathrm{~h}$ after transfection, then treated for the specified amounts of time with $\mathrm{CHX}$. Bottom: means of ataxin-3 signal quantified from western blots on the top and other similar, independent experiments. P-values are from Student's $t$-tests comparing protein levels of K-null ataxin-3 with the WT version of the protein. Error bars: s.d. $N=6$ independently conducted experiments.

Because ataxin-3 binds to proteins that associate with the proteasome, we examined whether UbS2 or the VCP-binding site regulates its stability. For the experiments described in Fig. 3a-f, we utilized ataxin- 3 constructs that do not contain lysine residues to eliminate any potentially confounding effects from ubiquitination at this stage of our study.

Surprisingly, mutating UbS2 (denoted as UbS2*; mutation is W87A unless noted otherwise) markedly reduces ataxin-3 steadystate protein levels in cultured mammalian cells (Fig. 3a). Mutating the VCP-binding site (denoted as $\mathrm{VCP}^{\star}$ ) does not consistently alter steady-state protein levels. In most experimental repeats, we did not detect a difference in the steady-state levels of K-null ataxin-3 with the VCP-binding site mutated compared with versions with intact VCP binding, as shown in Fig. 3a. There were a few instances where the levels of ataxin- 3 with mutated VCP binding were lower, as summarized by the quantification of multiple blots in Fig. 3a, but this difference did not reach statistical significance. Mutating both UbS2 and the VCP-binding site has an effect similar to that observed when only UbS2 is mutated (Fig. 3a). The studies in Fig. 3a were conducted in HeLa cells. A similar effect is observed in HEK-293 cells (Supplementary Fig. 3a).

The effect from mutating UbS2 does not result from differences at the mRNA level, as those levels are not significantly different (Fig. 3b). However, the difference in steady-state levels depends on the proteasome. Inhibiting proteasomal activity with MG132 equalizes the levels of ataxin-3 protein with mutated UbS2 to those of ataxin-3 with intact UbS2 (Fig. 3c; Supplementary Fig. 3b). Two other amino-acid residues important for UbS2 are tyrosine $(\mathrm{Y})$ at position 27 and phenylalanine $(\mathrm{F})$ at position $28^{21,22}$. Mutating those amino acids also leads to reduced steadystate levels of ataxin-3 protein in cultured cells (Fig. 3d).

Next, we examined the turnover rate of ataxin-3 with intact or mutated UbS2 by using CHX-based pulse-chase assays. As shown 
a
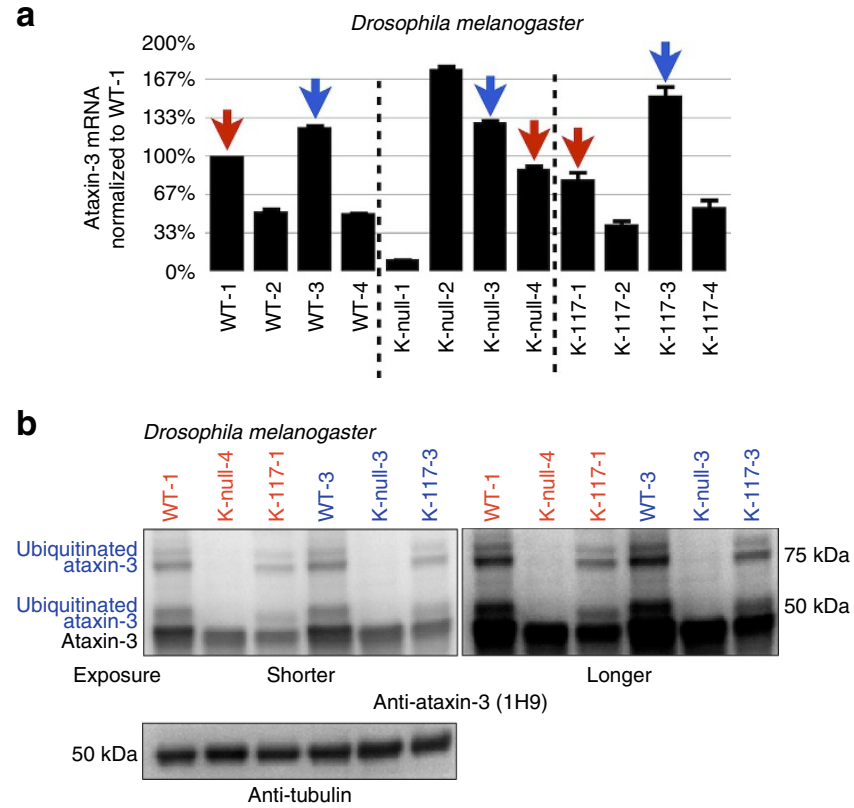

Figure 2 | Lysine-less ataxin-3 does not accumulate in vivo in Drosophila. (a) Selection of transgenic fly lines that express similar levels of ataxin-3 variants. Quantitative reverse transcriptase ( $q R T$ )-PCR results from whole flies expressing the versions of UAS-ataxin-3 noted in the panel. At least five flies were used per genotype per experiment. Driver was sqh-Gal4 (ubiquitous expression ${ }^{31,32}$ ). All flies were heterozygous for UAS-ataxin-3 and sqh-Gal4 transgenes. Red and blue arrows: lines that we chose for western blotting shown in panel $\mathbf{b}$. Experiment performed independently in triplicate. Shown are mean ataxin-3 mRNA levels normalized to WT-1. Error bars: s.d. Flies were 1-3 days old. (b) Western blots from whole-fly lysates expressing the indicated UAS-ataxin-3 constructs based on qRT-PCR data from panel a. Driver was sqh-Gal4. All flies were heterozygous for UAS-ataxin-3 and sqh-Gal4 transgenes, as in panel a. Ten or more flies per genotype were homogenized. Blots are representative of experiments performed independently in triplicate, with similar results. Flies were 1-3 days old. WT, wild-type.

in Fig. 3e, ataxin-3 protein with mutated UbS2 is turned over markedly more rapidly than ataxin-3 with intact UbS2. Mutating only the VCP-binding site does not significantly affect turnover (Fig. 3f). CHX-based analyses are consistent with steady-state examinations, indicating that UbS2 inhibits ataxin-3 degradation in cells, while VCP binding does not appear to have a significant effect. On the basis of these findings, we focus the rest of the studies in this report on UbS2.

As already stated, for experiments in Fig. 3a-f we used K-null ataxin-3 that contains intact or mutated UbS2. We next investigated whether the protein levels of ataxin-3 with all of its lysines present are also regulated by UbS2. Mutating UbS2 in an otherwise normal ataxin-3 markedly reduces its steady-state protein levels (Fig. 3g) and turnover (Fig. 3h), similar to what we observed with non-ubiquitinatable ataxin-3. Collectively, these results from mammalian cell culture indicate that UbS2 inhibits the proteasomal turnover of non-pathogenic ataxin-3.

UIMs of ataxin-3 have an opposite effect to UbS2 on turnover. For ataxin-3 to be degraded by the proteasome, it needs to come into contact with this cellular machinery. One possibility through which this interaction could occur would be through ataxin-3 ubiquitination. However, our results argue against ubiquitination being necessary for ataxin-3 degradation (Figs 1 and 2). Another mechanism that could bridge ataxin- 3 to the proteasome could be the poly-ubiquitin binding UIMs of this polyQ protein, which could bind ubiquitinated proteins targeted for the proteasome.

We mutated a conserved serine residue in each UIM of nonpathogenic ataxin-3 into an alanine, disrupting its interaction with ubiquitin chains ${ }^{26,29,38}$. Results in Fig. 4 indicate that the UIMs regulate the turnover of ataxin-3. Mutating all three UIMs counteracts the degradative effect of UbS2 mutation both at the steady-state level (Fig. 4a; Supplementary Fig. 4) and turnover rate (Fig. 4b). We conclude that the UIMs of ataxin-3 have a positive effect on its cellular turnover.

Rad23 regulates ataxin-3 protein levels in mammalian cells. Two proteins that bind ataxin-3 directly at UbS2 are Rad23A and $\operatorname{Rad} 23 \mathrm{~B}^{21,23}$. Therefore, we examined whether $\operatorname{Rad} 23 \mathrm{~A} / \mathrm{B}$ affect ataxin-3 protein levels in cultured cells by using RNAi. Knockdown of endogenous Rad23A or Rad23B through different, non-overlapping short interfering RNA constructs noticeably decreases levels of $\operatorname{Rad} 23 \mathrm{~A}$ or Rad23B protein. Their reduction is concomitant with a statistically significant reduction in the levels of endogenous ataxin-3 protein (Fig. 4c). Simultaneous knockdown of Rad23A and Rad23B has an even stronger effect in reducing normal, endogenous ataxin-3 protein levels (Fig. 4c). Together, these RNAi-based data are indicative of a protective role from $\operatorname{Rad} 23 \mathrm{~A}$ and $\operatorname{Rad} 23 \mathrm{~B}$ on ataxin-3.

UbS2 regulates normal ataxin-3 protein levels in Drosophila. Data in Figs 3 and 4 make the case that UbS2 of ataxin-3 is important for its stability in cultured cells. Next, we examined the significance of UbS2 in ataxin-3 protein levels in Drosophila. We generated new transgenic fly lines that express non-pathogenic ataxin-3 with mutated UbS2 and selected lines that express WT and $\mathrm{UbS2}^{\star}$ ataxin- 3 transgenes at similar mRNA levels, as well as ones that express the UbS2* variant more strongly (Fig. 5a). As shown in Fig. $5 b$, the protein levels of $\mathrm{UbS}^{*}$ ataxin-3 are markedly lower than those of WT ataxin-3 that is expressed similarly at the mRNA level. Even in transgenic lines where the mRNA levels of UbS2* ataxin-3 are approximately sixfold higher than WT ataxin-3, those protein levels do not quite approach the levels of normal ataxin-3. These data from an intact organism are consistent with our findings from cultured cells, and collectively support our overall conclusion that UbS2 regulates ataxin-3 protein levels by inhibiting its degradation.

UbS2 and Rad23 regulate pathogenic ataxin-3 in Drosophila. Finally, we examined whether mutating UbS2 on pathogenic ataxin-3 affects its stability (pathogenic variant denoted as SCA3; polyQ length of 81 for the intact version and 84 for UbS2*; these constructs contains all lysine residues). We found that mutating UbS2 of SCA3-causing ataxin-3 reduces its steady-state levels in cultured mammalian cells (Fig. 6a). Just as we observed with normal ataxin-3 (Fig. 3g,h), mutating UbS2 enhances the turnover rate of pathogenic ataxin-3 in cells (Fig. 6b). Also, proteasomal inhibition diminishes the marked difference in protein levels between ataxin- 3 versions that have an intact or mutated UbS2 (Fig. 6c). Together, these data indicate that the proteasomal turnover of pathogenic ataxin-3 in cells is also regulated by UbS2.

Because knockdown of Rad23A/B leads to lower levels of ataxin-3 protein in cultured cells (Fig. 4c), we reasoned that reducing Rad23 levels in Drosophila should suppress degeneration caused by its pathogenic version by lowering the levels of the polyQ protein. Whereas humans have separate genes for Rad23A and Rad23B, flies appear to have one gene for $\operatorname{Rad} 23$ (refs 21,23,24,39).

We utilized a transgenic fly line generated by the Bonini lab that expresses a full-length version of pathogenic ataxin-3 with 84 
a
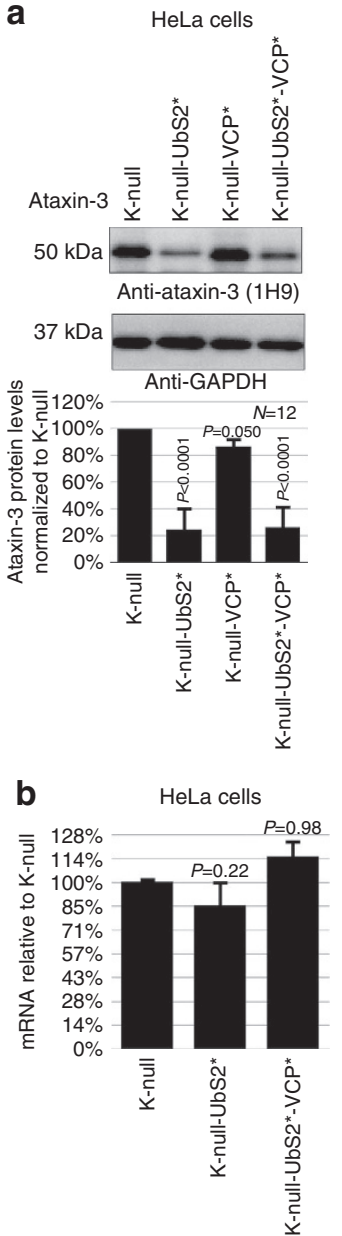

C

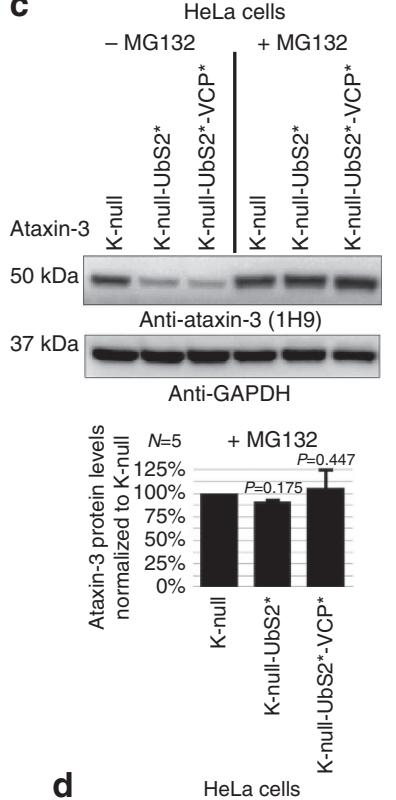

d

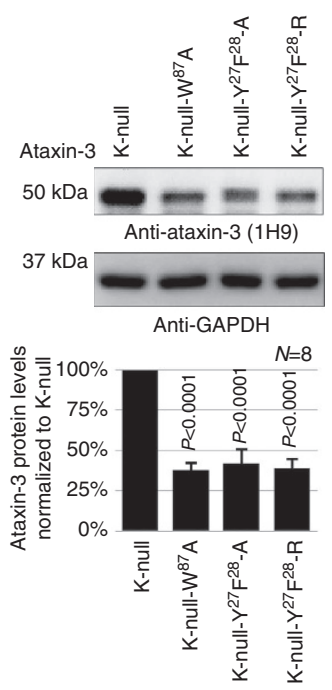

e
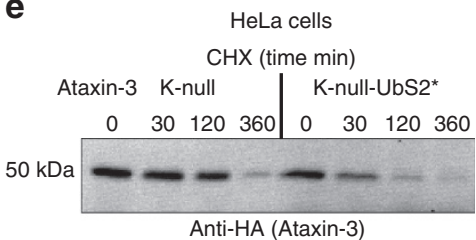

$50 \mathrm{kDa}$

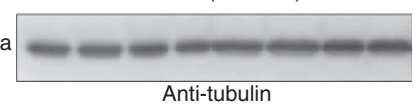

Anti-tubulin

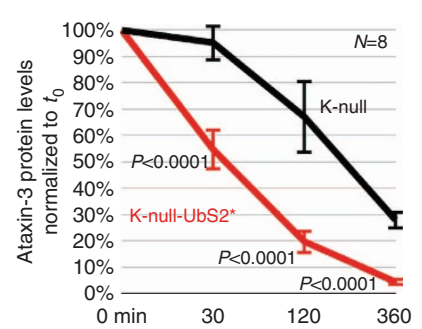

g
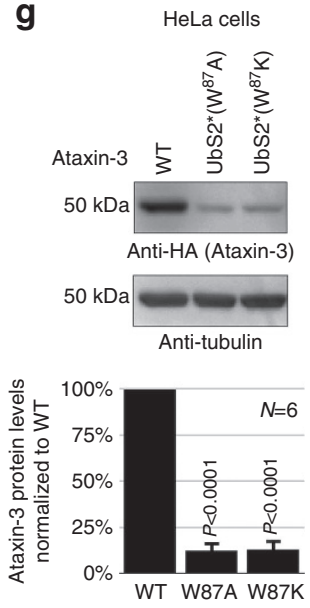

f

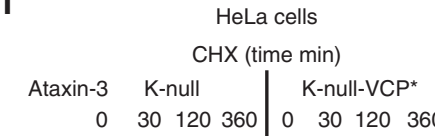

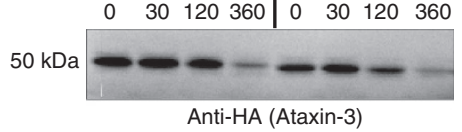

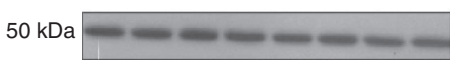

Anti-tubulin

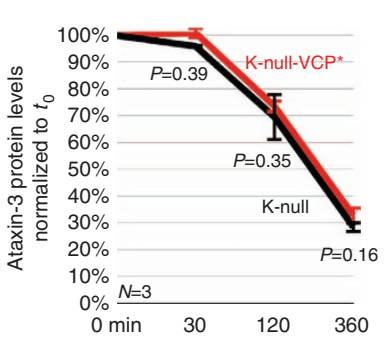

h

HeLa cells

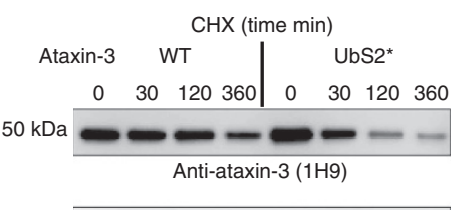

$50 \mathrm{kDa}$
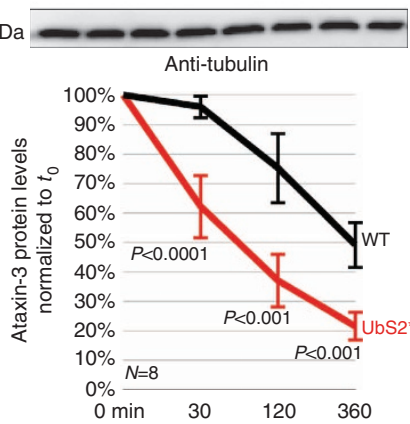

Figure 3 | UbS2 of ataxin-3 regulates its degradation in cells. (a) Top: western blots of whole-cell lysates. HeLa cells were transfected as indicated and harvested $24 \mathrm{~h}$ later. Bottom: means of ataxin-3 signal quantified from blots on the top and other similar experiments. Error bars: s.d. (b) Quantitative reverse transcriptase-PCR of HeLa cells expressing the indicated constructs. Endogenous control: GAPDH. $N=3$ independently conducted experiments. Shown are mean ataxin-3 mRNA levels \pm s.d. $(\mathbf{c}, \mathbf{d})$ Top: western blots of whole-cell lysates of HeLa cells transfected as indicated, treated or not with MG132 $(4 \mathrm{~h}, 15 \mu \mathrm{M}) 48 \mathrm{~h}$ later and harvested. Bottom: means of ataxin-3 signal quantified from blots on the top and other similar experiments. Error bars: s.d. (e,f) Top: HeLa cells were transfected as indicated and $24 \mathrm{~h}$ later were treated with $\mathrm{CHX}$ for the specified amounts of time. For panel e, to have comparable protein amounts of both ataxin-3 versions at time 0 min, we transfected three times more K-null-UbS2* construct than K-null, which was supplemented with empty vector to equate total DNA per group. Western blots of whole-cell lysates. Bottom: means of ataxin-3 signal quantified from blots on the top and other independent experiments. Error bars: s.d. (g) Top: western blots of whole-cell lysates. Two different mutations were used to disrupt UbS2: W87A and W87K, with similar results. Bottom: means of ataxin-3 signal quantified from blots on the top and other independent experiments. Error bars: s.d. (h) Top: HeLa cells were transfected as indicated and $24 \mathrm{~h}$ later were treated with $\mathrm{CHX}$ for the specified time points.

Three times more ataxin-3-UbS2* construct was transfected than ataxin-3-WT to have comparable protein levels at time 0 min. Bottom: means of ataxin-3 signal quantified from blots on the top and other independent experiments. Error bars: s.d. For panels $\mathbf{a}$-d and $\mathbf{g}, P$-values are from analysis of variance with Tukey's post hoc correction comparing the various versions of ataxin-3 with their panel controls. For panels $\mathbf{e}, \mathbf{f}$ and $\mathbf{h}, P$-values are from Student's $t$-tests. $N$ of independently repeated experiments is specified in panels.

polyQ repeats and has intact UbS2, VCP-binding site and $\mathrm{UIMs}^{6}$. First, we examined ataxin-3-SCA3 protein levels in fly eyes when Rad23 levels are reduced by either knocking down this gene through RNAi, or by using a chromosomal deletion in the genomic area that contains Rad23 (line Df(4)ED6369). This deficiency deletes chromosomal segment 102A1-102C1, which includes Rad23 at locus 102B3; these flies are hemizygous viable.
As shown in western blots in Fig. 6d, knocking down Rad23 or removing one copy of this gene leads to lower levels of ataxin-3 in Drosophila eyes. Knocking down other proteins related to ubiquitin (ubiquilin, nedd8 or SUMO, all of which reportedly interact with ataxin-3 (ref. 1)) does not reduce ataxin-3 protein levels in vivo (Supplementary Fig. 5), indicating a specific role for Rad23 in regulating ataxin-3. 
a

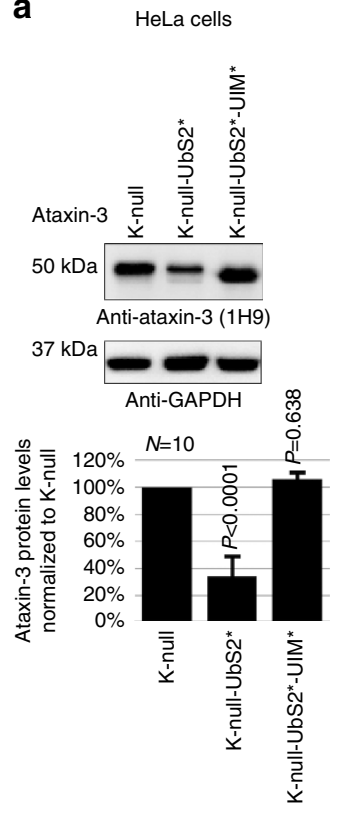

b

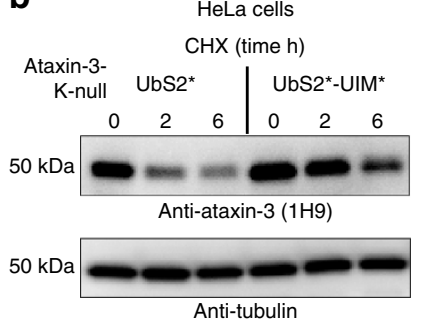

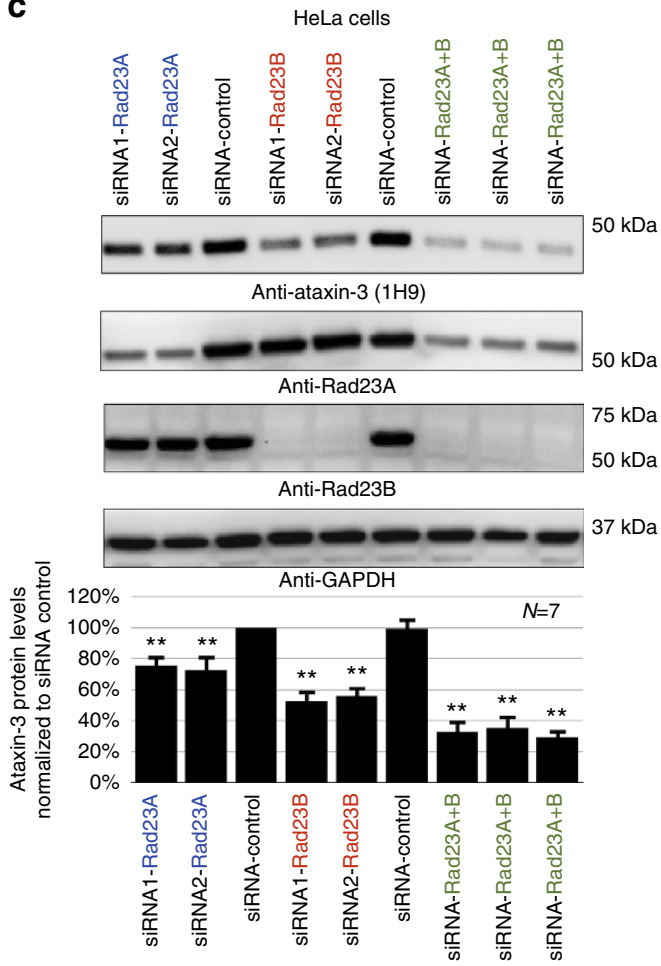

Figure 4 | UIMs of ataxin-3 oppose the effect of UbS2 mutation. (a) Top: HeLa cells were transfected as indicated and harvested $48 \mathrm{~h}$ later. Western blots from whole-cell lysates. Bottom: means of ataxin-3 signal quantified from blots on the top and other similar experiments. $P$-values are from analysis of variance (ANOVA) with Tukey's post hoc correction comparing K-null ataxin-3 with UbS2 mutated, and K-null ataxin-3 with UbS2 and UIMs mutated to K-null ataxin-3 with intact domains. Error bars: s.d. $N=10$ independently conducted experiments. (b) Top: HeLa cells were transfected with the indicated constructs. Three times more UbS2* DNA was used than UbS2*-UIM* to begin with approximately the same amount of protein at time $\mathrm{O}$ h. $\mathrm{CHX}$ was added to cells $24 \mathrm{~h}$ post transfection for the specified time points. Western blots of whole-cell lysates. Bottom: means of ataxin-3 signal quantified from blots on the top and other similar experiments. P-values are from Student's $t$-tests comparing ataxin-3 with UbS2 mutated with ataxin-3 with UbS2 and UIMs mutated. Error bars: s.d. $N=6$ independently conducted experiments. (c) Top: HeLa cells were transfected with the indicated short interfering RNA (siRNA) constructs to knock down endogenous Rad23A, endogenous Rad23B or both, and harvested $48 \mathrm{~h}$ later. Shown are western blots of whole-cell lysates. siRNA control: scramble controls. Bottom: means of ataxin-3 signal quantified from blots on the top and other similar experiments. $P$-values of $<0.01$ are indicated by '^*', and are from ANOVA/Tukey comparing the levels of ataxin-3 protein in RNAi lanes with those in scramble control. Error bars: s.d. $N=7$ independently conducted experiments.

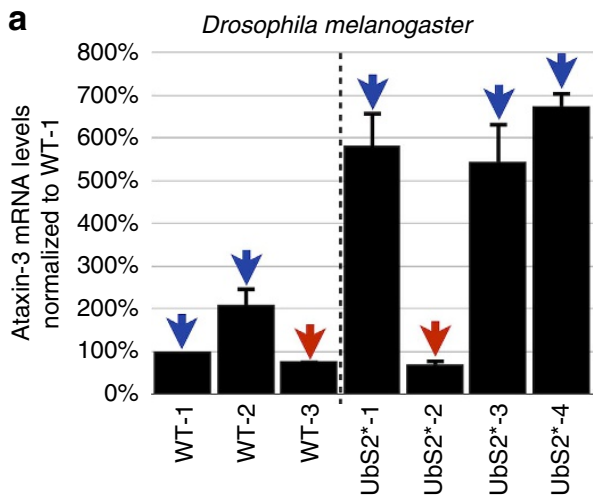

b
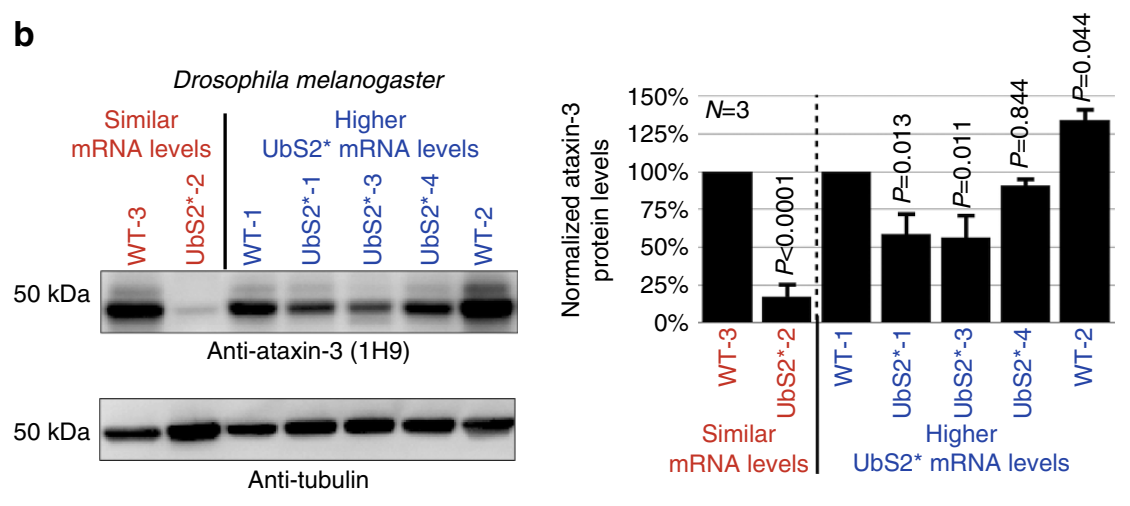

Figure 5 | UbS2 mutation leads to reduced ataxin-3 protein levels in Drosophila. (a) Quantitative reverse transcriptase (qRT)-PCR results from whole flies expressing the noted versions of UAS-ataxin-3 driven by sqh-Gal4. All flies were heterozygous for UAS-ataxin-3 and sqh-Gal4 transgenes. Red arrows: wild-type (WT) and UbS2* lines that have comparable ataxin-3 mRNA levels. Blue arrows: UbS2* lines that have markedly higher ataxin-3 mRNA levels than WT versions. Experiment performed independently in triplicate, utilizing at least five flies per genotype per experiment. Shown are mean ataxin-3 mRNA levels normalized to WT-1. Error bars: s.d. (b) Left: western blots from whole flies based on qRT-PCR results from a. At least five flies were homogenized per genotype. Driver was sqh-Gal4. All flies were heterozygous for UAS-ataxin-3 and sqh-Gal4 transgenes, as in a. Note that for this blot 1.5 times more lysate was loaded for the line that expresses UbS2*-2 to enable visualization of ataxin-3 protein in this line by western blotting without saturating the signal from other lysates. Right: means of ataxin-3 signal quantified from blots on the left and other independent experiments. $P$-values are from the Student's $t$-test (WT-3 and UbS2*-2) and analysis of variance/Tukey (the other lines). Error bars: s.d. $N=3$ independently conducted experiments. Flies were 1-3 days old. 
a

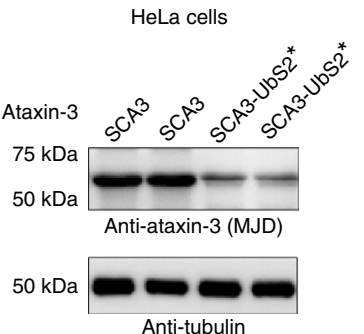

b
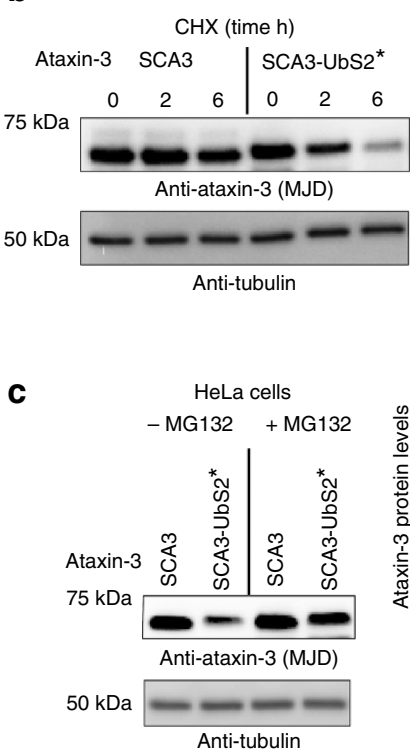
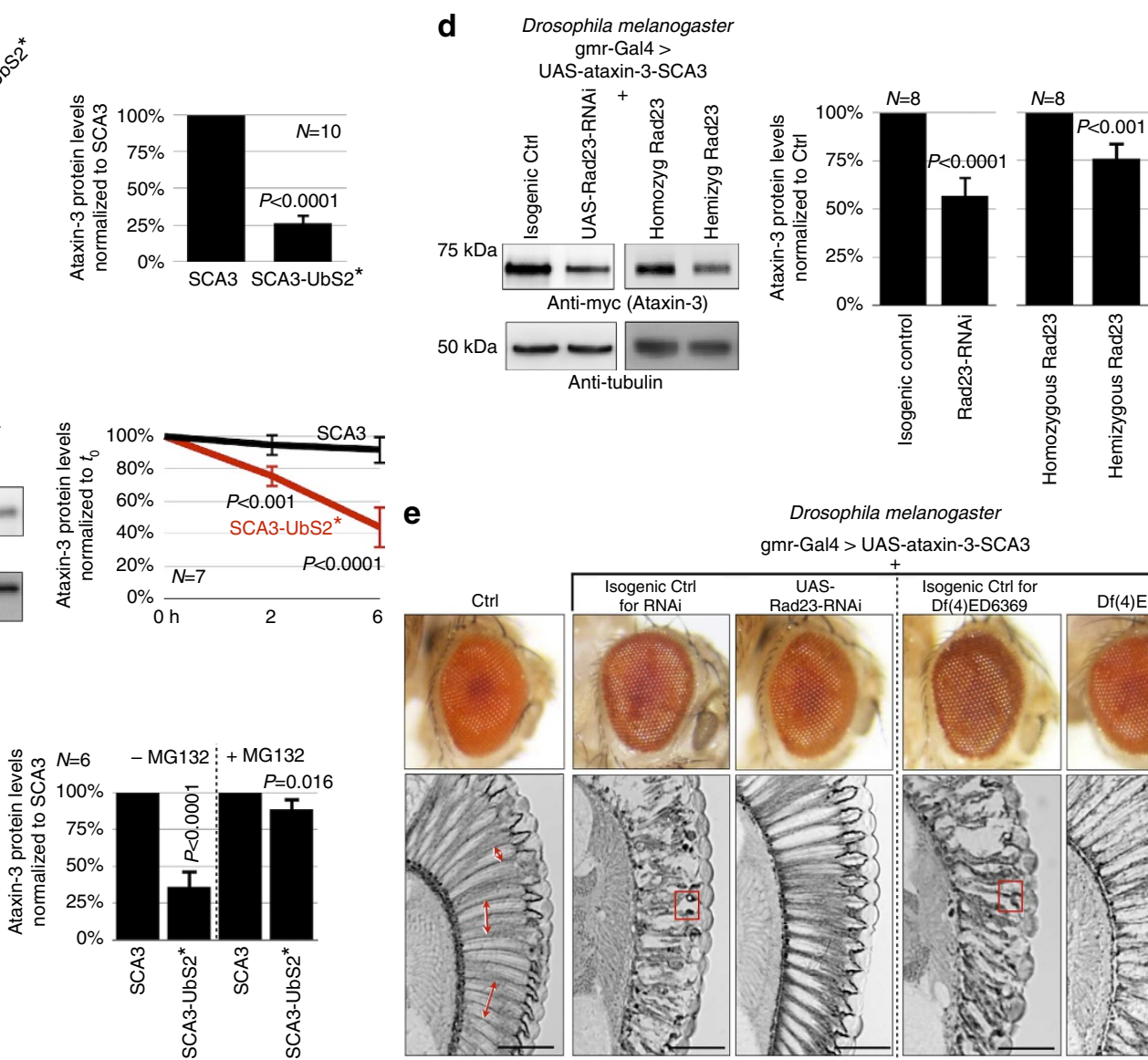

Drosophila melanogaster

gmr-Gal4 > UAS-ataxin-3-SCA3

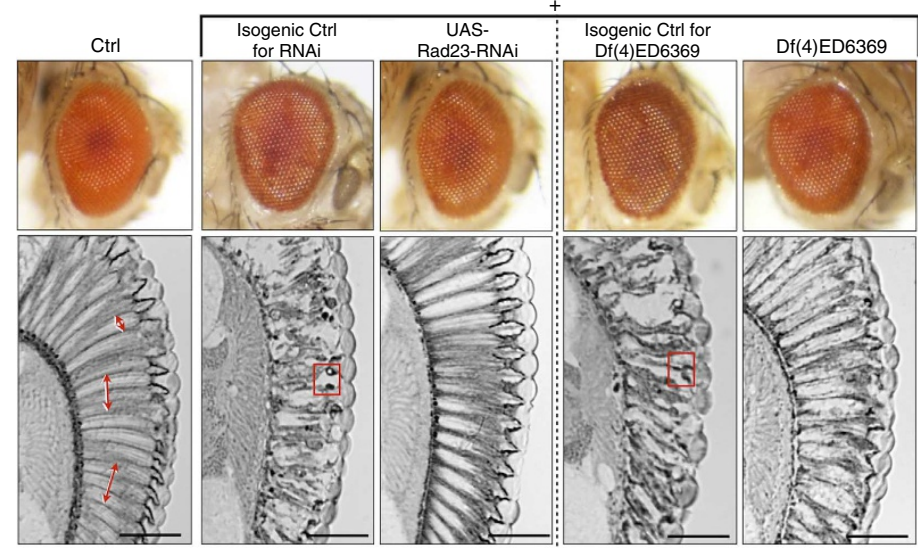

Figure 6 | Turnover of pathogenic ataxin-3 protein is also regulated by UbS2. (a,c) Left: western blots from HeLa cells transfected as indicated and treated or not with MG132 $(4 \mathrm{~h}, 15 \mu \mathrm{M}) 24 \mathrm{~h}$ after transfection. Right: means of ataxin-3 signal quantified from blots on the left and other similar experiments. $P$-values are from Student's $t$-tests. Error bars: s.d. $N$ of independently conducted experiments is provided in the respective panels.

(b) Left: western blots of HeLa cells transfected as indicated and treated with $\mathrm{CHX} 24 \mathrm{~h}$ later. Right: means of ataxin-3 signal quantified from blots on the left and other similar experiments. $P$-values are from Student's $t$-tests. Error bars: s.d. $N=7$ independently conducted experiments. (d) Left: western blots from at least 10 dissected fly heads for each indicated group. Right: means of ataxin-3 signal quantified from blots on the left and other similar experiments. P-values are from Student's $t$-tests. Error bars: s.d. $N=8$ independent experiments. Flies were 1-2 days old. All flies were heterozygous for UAS-ataxin-3, UAS-Rad23-RNAi (where indicated) and the gmr-Gal4 driver. (e) External photos and internal sections of fly eyes expressing UAS-ataxin-3-SCA3 in the isogenic background of the UAS-RNAi line targeting Rad23 (2nd column), with UAS-RNAi targeting Rad23 (3rd column), in the isogenic background of Rad23 deficiency (4th column, which is the control for column 5; see main text for description) or in the presence of one copy of Rad23 (deficiency line; 5th column). Driver: gmr-Gal4. Double-headed arrows: ommatidial boundaries. Boxes: proteinaceous inclusions that contain ataxin-3-SCA3 (ref. 6). Control in the 1st column contained only gmr-Gal4. The other lines were heterozygous for the gmr-Gal4 driver and UAS-transgenes. Images are representative of sections from at least six flies per group and experiments were conducted independently in triplicate with similar results. Flies were 14 days old. Scale bars in histological sections, $50 \mu \mathrm{m}$.

We then examined whether reduction of Rad23 suppresses degeneration caused by pathogenic ataxin-3. When expressed selectively in fly eyes, this ataxin-3 variant does not greatly impact the external eye compared with wild-type controls (Fig. 6e, compare columns I and II). However, retinal sections show marked degeneration (disruption and loss of the radial ommatidial array and less-defined inter-ommatidial boundaries) and the presence of densely staining structures/aggregates, which contain pathogenic ataxin-3 (ref. 6). As shown in Fig. 6e column III, knockdown of Rad23 markedly suppresses degeneration caused by pathogenic ataxin-3 in fly eyes. We recapitulated these findings from the RNAi targeting Rad23 with the deficiency line described above. When we express pathogenic ataxin-3 on a Rad23 hemizygous background, we again observe suppressed SCA3-dependent retinal degeneration (columns IV and V in Fig. 6e). Together, data in Fig. 6 indicate that pathogenic ataxin-3 protein is also regulated by UbS2 and $\operatorname{Rad} 23$.

\section{Discussion}

The degradation of most short-lived, abnormal and misfolded proteins in eukaryotic cells is accomplished by the ubiquitin-proteasome pathway ${ }^{20}$. In this pathway, proteins destined for the proteasome are tagged post-translationally with ubiquitin chains, which enable their recognition by proteasomeassociated ubiquitin-binding proteins. Once bound to the proteasome, these ubiquitinated substrates are deubiquitinated and ultimately degraded ${ }^{20}$. In our search to determine how the disease protein ataxin-3 is degraded, we collected evidence that the turnover of this polyQ protein does not require its own ubiquitination: ataxin-3 that does not become ubiquitinated is degraded similarly to its wild-type version in cells and does not accumulate in vivo in Drosophila.

According to a previous report, the E3 ubiquitin ligase Cterminus of Hsc70 Interacting Protein (CHIP) may ubiquitinate ataxin-3 as well as other polyQ proteins to target them for degradation ${ }^{40}$. Indeed, we previously showed that ataxin- 3 is 


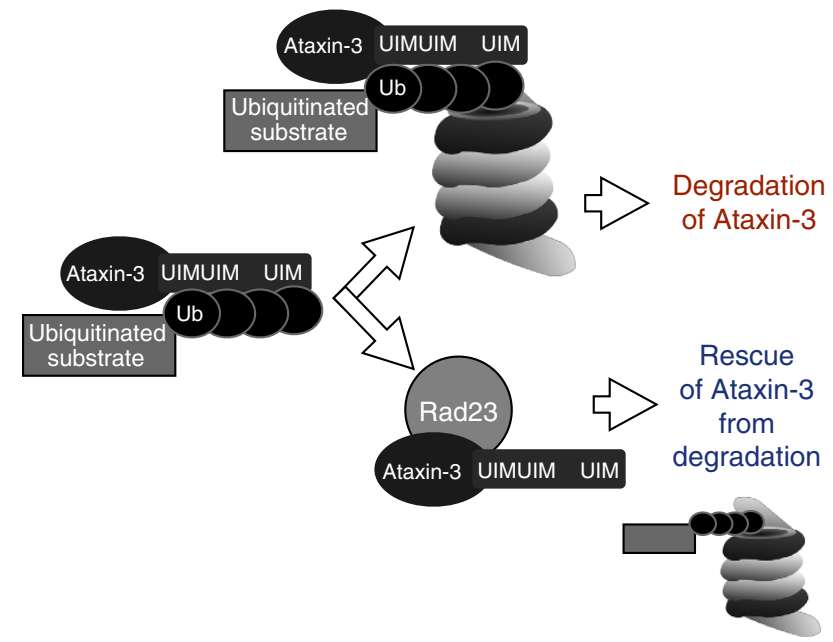

Figure 7 | Proposed model of ataxin-3 degradation. According to this model, ataxin-3 comes into physical contact with the proteasome by binding to ubiquitinated proteins through its UIMs in the C-terminal portion. Once at the proteasome, in the absence of interaction with Rad23A/B through UbS2, ataxin-3 is degraded by the proteasome. If UbS2 interacts with $\operatorname{Rad} 23 \mathrm{~A} / \mathrm{B}$, ataxin-3 is rescued from degradation, perhaps as a result of higher affinity for Rad23A/B compared with the proteasome.

ubiquitinated in mammalian cells and in Drosophila ${ }^{7,26-28}$, and that ubiquitination directly and markedly enhances its DUB activity ${ }^{7,26-28}$. However, recent work indicated that CHIP ubiquitinates ataxin-3 to modify its catalytic activity during the process of substrate ubiquitination, where ataxin-3 and CHIP cooperate to enhance CHIP substrate degradation ${ }^{26-28}$. In addition, CHIP knockout mice do not show accumulation of ataxin-3 protein, and knockdown of CHIP in Drosophila does not detectably impact ataxin-3 protein levels ${ }^{7}$. On the basis of these previously published findings and our present results, we propose that ubiquitination of ataxin-3 serves to regulate its DUB functions, rather than directly dictate its proteasomal turnover. This is not to say that ubiquitination of ataxin-3 might not enhance its turnover under some circumstances. What our results indicate is that ataxin-3 does not need to be ubiquitinated to be degraded. Other proteins have been reported to be degraded by the proteasome in the absence of their own ubiquitination, including $\mathrm{p} 21 / \mathrm{CiP} 1$ (ref. 41), calmodulin ${ }^{42}$, thymidylate synthase $^{43}$, tau $^{44}$ and so on ${ }^{45}$.

We found that ataxin-3 degradation is regulated by its UbS2. Mutation of this domain accelerates the turnover of ataxin-3 in cells and leads to markedly lower levels of this protein in vivo. The general structural organization of the catalytic domain of ataxin-3 appears to not be impacted by mutating residue W87 of UbS2, according to previously published nuclear magnetic resonance (NMR) studies ${ }^{21,23}$. The localization of full-length ataxin-3-UbS2* in cultured mammalian cells is not different from versions of this protein with intact UbS2. Full-length ataxin-3 with mutated UbS2 is capable of cleaving ubiquitin chains in vitro ${ }^{23}$, and this site is dispensable for the ubiquitinationdependent activation of ataxin-3 that we have reported previously $y^{23,26,27}$. On the basis of these findings, mutating UbS2 does not detrimentally impact ataxin-3's folding and localization, or abrogate its basic catalytic activities.

UbS2 binds directly to Rad23A/ $\mathrm{B}^{21-23}$. Knockdown of Rad23A and/or B in cultured cells leads to significantly lower levels of ataxin-3 protein. Importantly, Rad23 knockdown in Drosophila leads to lower levels of pathogenic ataxin-3 and ameliorates retinal degeneration caused by this protein. On the basis of our collective results, we propose a model for ataxin-3 degradation, whereby its UIMs bind ubiquitinated proteasome substrates and 'bring' this protein quality control DUB into the proximity of the degradative machinery. Once near the proteasome, the interaction of ataxin-3 with $\operatorname{Rad} 23$ prevents it from being degraded, perhaps because the direct binding of this polyQ protein to Rad23 is stronger than its association with the proteasome. If ataxin-3 does not bind $\operatorname{Rad} 23$, it is then degraded (Fig. 7).

Our finding that UbS2 controls ataxin-3 degradation is of critical importance to SCA3 therapeutics. The structure of the $\mathrm{N}$-terminal half of ataxin-3, including UbS2, has been solved by NMR spectroscopy, and its interaction with the ubiquitin-like domain of $\operatorname{Rad} 23$ has been modelled at this level ${ }^{21-23}$. We propose that this structural information be utilized to generate compounds that bind ataxin-3 at UbS2 and inhibit its interaction with $\operatorname{Rad} 23$ to increase the degradation rate of pathogenic ataxin-3 in SCA3 patients.

The work that we presented might be also of significance to diseases other than SCA3. Rad23A is reported to also interact directly with ataxin-7 (ref. 46), mutations in which cause SCA7 (ref. 4). It would be of interest to determine whether Rad23A inhibits the degradation of ataxin-7, similar to its effect on ataxin-3. Moreover, a recent study by Sacco et al. ${ }^{47}$ provided convincing evidence that ataxin-3 restricts the transcription of the tumour suppressor phosphatase and tensin homolog deleted on chromosome ten (PTEN). PTEN is considered a viable therapeutic target for various malignancies ${ }^{48,49}$. The mechanism of ataxin-3 degradation that we presented leads us to suggest that enhancing the turnover of this enzyme could conceivably be utilized for some cancer therapeutics: lowering ataxin-3 levels would be expected to increase the levels of PTEN.

Last, our findings suggest additional complexity in basic mechanisms of protein quality control. The general view of proteasome-associated proteins is that they enhance the degradation of client proteins with which they interact ${ }^{34,50,51}$. Our data propose that some proteasome-associated proteins, for example, Rad23, can also inhibit, not just enhance, protein turnover. This finding suggests that proteasome shuttles need not always function in one direction, and expands the overall understanding of how individual components perform during protein quality control.

\section{Methods}

Cell lines. HeLa and HEK-293 cells were purchased from ATCC and were grown in Dulbecco's modified Eagle's medium $+10 \%$ fetal bovine serum under conventional tissue culture conditions.

Constructs. Haemagglutinin (HA)-tagged ataxin-3 constructs are in pCDNA3.1-HA; this includes all non-pathogenic ataxin-3 variants. Mutations were generated on the wild-type ataxin-3 backbone using the QuikChange mutagenesis kit (Agilent). FLAG-tagged ataxin-3 constructs are in pFLAG6a (Sigma-Aldrich) ${ }^{25,26,29}$; this includes the pathogenic ataxin-3 variants. Mutations in these constructs were also conducted using the QuikChange mutagenesis kit (Agilent). Where noted, lysine residues in ataxin-3 were mutated into the similar but non-ubiquitinatable amino-acid arginine ${ }^{7,27}$. RNAi constructs targeting Rad23A or B in mammalian cells were purchased from Invitrogen. Catalog numbers: s11729, s11730, s11731 and s11733.

Transfections. Cells were transfected using Lipofectamine LTX (Invitrogen) as directed by the manufacturer for all constructs, except for short interfering RNA, where we used Lipofectamine RNAiMAX (Invitrogen).

SDS-PAGE. Cells were harvested for SDS-polyacrylamide gel electrophoresis (PAGE) in boiling 2\% SDS lysis buffer (50 mM Tris pH 6.8,2\% SDS, $10 \%$ glycerol and $100 \mathrm{mM}$ dithiothreitol $)^{25,29,52}$, boiled for $10 \mathrm{~min}$, centrifuged, loaded onto $10 \%$ or 4-20\% SDS-PAGE gels, electrophoresed at 150-180 V and transferred onto polyvinylidene difluoride membrane (Bio-Rad) for western blotting. 
Antibodies. Anti-ataxin-3 (MJD; rabbit polyclonal, 1:15,000 (ref. 53)), antiataxin-3 monoclonal (clone 1H9, mouse, 1:500-1:1,000; Millipore), anti-HA (rabbit polyclonal Y11, 1:500; Santa Cruz Biotech), anti-tubulin (mouse monoclonal T5168, 1:10,000; Sigma-Aldrich), anti-GAPDH (mouse monoclonal MAB374, 1:500; Millipore), anti-Rad23A (rabbit polyclonal YH62308, 1:5,000; Origene), anti-Rad23B (rabbit polyclonal A302-306A, 1:2,000; Bethyl Labs), peroxidaseconjugated secondary antibodies (goat anti-rabbit and goat anti-mouse, 1:5,000; Jackson Immunoresearch)

Western blotting and signal quantification. Western blots were imaged with a charge-coupled device-equipped VersaDoc 5000MP system (Bio-Rad) ${ }^{26,27,29}$ Quantification of signals from sub-saturated blots was conducted in the Quantity One software (Bio-Rad) with universal background subtraction. Signal from the protein of interest was normalized to its own loading control (tubulin or GAPDH). Experimental lanes were normalized to respective controls. Student's $t$-tests with two tails and analysis of variance with Tukey's post hoc correction were used for statistical comparisons, as specified in the figure legends. Full western blots for images in Figs 1-6 are shown in Supplementary Fig. 6.

Chemicals. CHX was purchased from A.G. Scientific, dissolved in ultra-pure water and used at final concentration of $50 \mu \mathrm{g} \mathrm{ml}^{-1}$. MG132 was purchased from A.G. Scientific, dissolved in DMSO and utilized at final concentrations specified in figure legends.

Drosophila lines. Common stocks and the line expressing pathogenic ataxin-3 (stock number 33610 with genotype $\mathrm{w}[1118]$; $\mathrm{P}\{\mathrm{w}[+\mathrm{mC}]=$ UAS-SCA3.flQ84.myc 7.2/MKRS) were purchased from the Bloomington Drosophila Stock Center. The UAS-RNAi line targeting Rad23 and its isogenic background were purchased from the Vienna Drosophila RNAi Center (transformants 30498 and 60000). The Rad23 deficiency line was purchased from Bloomington Drosophila Stock Center (stock number: 9422 with genotype w[1118]; Df(4)ED6369, $\mathrm{P}\left\{\mathrm{w}[+\mathrm{mW}\right.$. Scer $\backslash$ FRT.hs3 $]=3^{\prime}$.RS5 + 3.3'\}ED6369/l(4)102EFf[1]). Nonpathogenic UAS-ataxin-3 flies were generated by us, using ataxin-3 with a polyQ repeat of 22 residues cloned into the pUASt vector. Injections were done by the Duke University Model System Genomics into the $\mathrm{w}^{1118}$ background ${ }^{7}$. All fly lines were on a w[-] background. Flies were maintained in a diurnally controlled $25^{\circ} \mathrm{C}$ environment at $\sim 60 \%$ humidity. Whole flies or dissected heads were homogenized in boiling SDS lysis buffer (50 mM Tris pH 6.8,2\% SDS, $10 \%$ glycerol and $100 \mathrm{mM}$ dithiothreitol), sonicated, boiled for $10 \mathrm{~min}$, centrifuged to remove debris, loaded onto SDS-PAGE gels, electrophoresed and transferred onto polyvinylidene difluoride membrane for western blotting ${ }^{7,54}$. We used 5-10 whole flies or 10-15 heads per group, and $50 \mu \mathrm{l}$ lysis buffer per whole fly or $10 \mu \mathrm{l}$ per dissected head. Analysis of Drosophila samples was conducted in a blinded manner. Choice of sample size for Drosophila studies was based on common practices among fly labs. No samples were excluded from analyses.

Quantitative reverse transcriptase-PCR. Total RNA was extracted from cultured mammalian cells or 1-3-day-old adult flies using TRIzol reagent (Invitrogen). Extracted RNA was treated with TURBO DNAse (Ambion) to eliminate contaminating DNA. Reverse transcription was performed with the High Capacity Kit (ABI), and messenger RNA levels were quantified by using the PlusOne real-time quantitative system with fast SYBR green (ABI). rp49 and GAPDH were used as internal controls. Primers: rp49-F: $5^{\prime}$-AGATCGTGAAGAAGCGCACCAAG-3', rp49-R: 5'-CACCAGGAACTTCTTGAATCCGG-3'; GAPDH-F: 5'-GCTCAGAC ACCATGGGGAAGGT-3', GAPDH-R: $5^{\prime}$-GTGGTGCAGGAGGCATTGCTGA-3'; ataxin-3 in flies: AT3-F: 5'-GAATGGCAGAAGGAGGAGTTACTA-3', AT3-R: $5^{\prime}$-GACCCGTCAAGAGAGAATTCAAGT- $3^{\prime}$; ataxin-3 in mammalian cells: AT3-F: 5' -TTCCAGATTACGCTTCTAGAGGAT-3', AT3-R: 5'-TAGTAACTCC TCCTTCTGCCATTC- $3^{\prime}$

Fly histology. Whole flies with removed proboscises were fixed in $2 \%$ glutaraldehyde/2\% paraformaldehyde in Tris-buffered saline overnight. Fixed flies were then dehydrated in a series of $30,50,75$ and $100 \%$ ethanol and propylene oxide, embedded in Poly/Bed812 (Polysciences) and sectioned at $5 \mu \mathrm{m}$. Sections were stained with toluidine blue.

\section{References}

1. Costa Mdo, C. \& Paulson, H. L. Toward understanding Machado-Joseph disease. Prog. Neurobiol. 97, 239-257 (2012)

2. Todi, S. V., Williams, A. \& Paulson, H. L. in Molecular Neurology 1st edn (ed. Waxman, S. G.) (Academic Press, 2007)

3. Williams, A. J. \& Paulson, H. L. Polyglutamine neurodegeneration: protein misfolding revisited. Trends Neurosci. 31, 521-528 (2008)

4. Orr, H. T. \& Zoghbi, H. Y. Trinucleotide repeat disorders. Ann. Rev. Neurosci. 30, 575-621 (2007)

5. Reina, C. P., Zhong, X. \& Pittman, R. N. Proteotoxic stress increases nuclear localization of ataxin-3. Hum. Mol. Genet. 19, 235-249 (2010).
6. Warrick, J. M. et al. Ataxin-3 suppresses polyglutamine neurodegeneration in Drosophila by a ubiquitin-associated mechanism. Mol. Cell 18, 37-48 (2005).

7. Tsou, W.-L. et al. Ubiquitination regulates the neuroprotective function of the deubiquitinase ataxin-3 in vivo. J. Biol. Chem. 288, 34460-34469 (2013).

8. Pedersen, J. T. \& Heegaard, N. H. Analysis of protein aggregation in neurodegenerative disease. Anal. Chem. 85, 8254-8261 (2013).

9. Rodriguez-Lebron, E., Gouvion, C. M., Moore, S. A., Davidson, B. L. \& Paulson, H. L. Allele-specific RNAi mitigates phenotypic progression in a transgenic model of Alzheimer's disease. Mol. Ther. 17, 1563-1573 (2009).

10. Alves, S. et al. Silencing ataxin-3 mitigates degeneration in a rat model of Machado-Joseph disease: no role for wild-type ataxin-3? Hum. Mol. Genet. 19, 2380-2394 (2010).

11. Alves, S. et al. Striatal and nigral pathology in a lentiviral rat model of Machado-Joseph disease. Hum. Mol. Genet. 17, 2071-2083 (2008).

12. Williams, A. J., Knutson, T. M., Colomer Gould, V. F. \& Paulson, H. L. In vivo suppression of polyglutamine neurotoxicity by C-terminus of Hsp70interacting protein (CHIP) supports an aggregation model of pathogenesis. Neurobiol. Dis. 33, 342-353 (2009).

13. Harper, S. Q. et al. RNA interference improves motor and neuropathological abnormalities in a Huntington's disease mouse model. Proc. Natl Acad. Sci. USA 102, 5820-5825 (2005).

14. Xia, H. et al. RNAi suppresses polyglutamine-induced neurodegeneration in a model of spinocerebellar ataxia. Nat. Med. 10, 816-820 (2004).

15. Tsou, W. L., Soong, B. W., Paulson, H. L. \& Rodriguez-Lebron, E. Splice isoform-specific suppression of the Cav2.1 variant underlying spinocerebellar ataxia type 6. Neurobiol. Dis. 43, 533-542 (2011).

16. Alves, S. et al. Allele-specific RNA silencing of mutant ataxin-3 mediates neuroprotection in a rat model of Machado-Joseph disease. PLoS ONE 3, e3341 (2008).

17. Bove, J., Martinez-Vicente, M. \& Vila, M. Fighting neurodegeneration with rapamycin: mechanistic insights. Nat. Rev. Neurosci. 12, 437-452 (2011).

18. do Carmo Costa, M. et al. Toward RNAi therapy for the polyglutamine disease Machado-Joseph disease. Mol. Ther. 21, 1898-1908 (2013).

19. Nobrega, C. et al. Silencing mutant ataxin-3 rescues motor deficits and neuropathology in machado-joseph disease transgenic mice. PLOS ONE 8, e52396 (2013)

20. Heride, C., Urbe, S. \& Clague, M. J. Ubiquitin code assembly and disassembly. Curr. Biol. 24, 215-220 (2014)

21. Nicastro, G. et al. Josephin domain of ataxin-3 contains two distinct ubiquitin binding sites. Biopolymers 91, 1203-1214 (2009).

22. Nicastro, G. et al. The solution structure of the Josephin domain of ataxin-3: structural determinants for molecular recognition. Proc. Natl Acad. Sci. USA 102, 10493-10498 (2005).

23. Nicastro, G. et al. Understanding the role of the Josephin domain in the PolyUb binding and cleavage properties of ataxin-3. PLoS ONE 5, e12430 (2010).

24. Wang, G., Sawai, N., Kotliarova, S., Kanazawa, I. \& Nukina, N. Ataxin-3, the MJD1 gene product, interacts with the two human homologs of yeast DNA repair protein RAD23, HHR23A and HHR23B. Hum. Mol. Genet. 9, 1795-1803 (2000).

25. Todi, S. V. et al. Cellular turnover of the polyglutamine disease protein ataxin-3 is regulated by its catalytic activity. J. Biol. Chem. 282, 29348-29358 (2007).

26. Todi, S. V. et al. Ubiquitination directly enhances activity of the deubiquitinating enzyme ataxin-3. EMBO J. 28, 372-382 (2009).

27. Todi, S. V. et al. Activity and cellular functions of the deubiquitinating enzyme and polyglutamine disease protein ataxin-3 are regulated by ubiquitination at lysine 117. J. Biol. Chem. 285, 39303-39313 (2010).

28. Scaglione, K. M. et al. Ube $2 \mathrm{w}$ and ataxin-3 coordinately regulate the ubiquitin ligase CHIP. Mol. Cell 43, 599-612 (2011).

29. Winborn, B. J. et al. The deubiquitinating enzyme ataxin-3, a polyglutamine disease protein, edits Lys63 linkages in mixed linkage ubiquitin chains. J. Biol. Chem. 283, 26436-26443 (2008).

30. Brand, A. H. \& Perrimon, N. Targeted gene expression as a means of altering cell fates and generating dominant phenotypes. Development 118, 401-415 (1993).

31. Todi, S. V., Franke, J. D., Kiehart, D. P. \& Eberl, D. F. Myosin VIIA defects, which underlie the Usher $1 \mathrm{~B}$ syndrome in humans, lead to deafness in Drosophila. Curr. Biol. 15, 862-868 (2005).

32. Franke, J. D., Boury, A. L., Gerald, N. J. \& Kiehart, D. P. Native nonmuscle myosin II stability and light chain binding in Drosophila melanogaster. Cell Motil. Cytoskeleton 63, 604-622 (2006).

33. Buchberger, A., Bukau, B. \& Sommer, T. Protein quality control in the cytosol and the endoplasmic reticulum: brothers in arms. Mol. Cell 40, 238-252 (2010).

34. Dantuma, N. P., Heinen, C. \& Hoogstraten, D. The ubiquitin receptor Rad23: at the crossroads of nucleotide excision repair and proteasomal degradation. DNA Repair (Amst) 8, 449-460 (2009).

35. Boeddrich, A. et al. An arginine/lysine-rich motif is crucial for $\mathrm{VCP} / \mathrm{p} 97-$ mediated modulation of ataxin-3 fibrillogenesis. EMBO J. 25, 1547-1558 (2006). 
36. Morreale, G., Conforti, L., Coadwell, J., Wilbrey, A. L. \& Coleman, M. P. Evolutionary divergence of valosin-containing protein/cell division cycle protein 48 binding interactions among endoplasmic reticulum-associated degradation proteins. FEBS J. 276, 1208-1220 (2009).

37. Zhong, X. \& Pittman, R. N. Ataxin-3 binds VCP/p97 and regulates retrotranslocation of ERAD substrates. Hum. Mol. Genet. 15, 2409-2420 (2006).

38. Shoesmith Berke, S. J., Chai, Y., Marrs, G. L., Wen, H. \& Paulson, H. L. Defining the role of ubiquitin interacting motifs in the polyglutamine disease protein, ataxin-3. J. Biol. Chem. 280, 32026-32034 (2005).

39. A database of Drosophila genes \& genomes. Flybase.org (2014).

40. Jana, N. R. et al. Co-chaperone CHIP associates with expanded polyglutamine protein and promotes their degradation by proteasomes. J. Biol. Chem. 280, 11635-11640 (2005).

41. Sheaff, R. J. et al. Proteasomal turnover of p21Cip1 does not require p21Cip1 ubiquitination. Mol. Cell 5, 403-410 (2000).

42. Tarcsa, E., Szymanska, G., Lecker, S., O’Connor, C. M. \& Goldberg, A. L. $\mathrm{Ca} 2+-$ free calmodulin and calmodulin damaged by in vitro aging are selectively degraded by $26 \mathrm{~S}$ proteasomes without ubiquitination. J. Biol. Chem. 275, 20295-20301 (2000).

43. Forsthoefel, A. M., Pena, M. M., Xing, Y. Y., Rafique, Z. \& Berger, F. G. Structural determinants for the intracellular degradation of human thymidylate synthase. Biochemistry 43, 1972-1979 (2004).

44. Blair, L. J. et al. Accelerated neurodegeneration through chaperone-mediated oligomerization of tau. J. Clin. Invest. 123, 4158-4169 (2013).

45. Hoyt, M. A. \& Coffino, P. Ubiquitin-free routes into the proteasome. Cell. Mol. Life Sci. 61, 1596-1600 (2004).

46. Lim, J. et al. A protein-protein interaction network for human inherited ataxias and disorders of Purkinje cell degeneration. Cell 125, 801-814 (2006).

47. Sacco, J. J. et al. The deubiquitylase Ataxin-3 restricts PTEN transcription in lung cancer cells. Oncogene 33, 4265-4272 (2014).

48. Song, M. S., Salmena, L. \& Pandolfi, P. P. The functions and regulation of the PTEN tumour suppressor. Nat. Rev. Mol. Cell. Biol. 13, 283-296 (2012).

49. Leslie, N. R. \& Foti, M. Non-genomic loss of PTEN function in cancer: not in my genes. Trends Pharmacol. Sci. 32, 131-140 (2011).

50. Claessen, J. H., Kundrat, L. \& Ploegh, H. L. Protein quality control in the ER: balancing the ubiquitin checkbook. Trends Cell Biol. 22, 22-32 (2012).
51. Yamanaka, K., Sasagawa, Y. \& Ogura, T. Recent advances in p97/VCP/Cdc48 cellular functions. Biochim. Biophys. Acta 1823, 130-137 (2012).

52. Blount, J. R., Burr, A. A., Denuc, A., Marfany, G. \& Todi, S. V. Ubiquitinspecific protease 25 functions in Endoplasmic Reticulum-associated degradation. PLoS ONE 7, e36542 (2012).

53. Paulson, H. L. et al. Intranuclear inclusions of expanded polyglutamine protein in spinocerebellar ataxia type 3. Neuron 19, 333-344 (1997).

54. Tsou, W.-L. et al. Systematic analysis of the physiological importance of deubiquitinating enzymes. PLoS ONE 7, e43112 (2012).

55. Scaglione, K. M. et al. The ubiquitin-conjugating enzyme (E2) Ube2w ubiquitinates the $\mathrm{N}$ terminus of substrates. J. Biol. Chem. 288, 18784-18788 (2013).

\section{Acknowledgements}

This work was funded by R00NS064097 and R01NS086778 to S.V.T. from NINDS, by awards to S.V.T. and W.-L.T. from the National Ataxia Foundation, by a T32 training slot to A.A.B. (CA009531) and by R00NS073936 to K.M.S. from NINDS.

\section{Author contributions}

J.R.B., W.-L.T., K.M.S. and S.V.T. designed the experiments. J.R.B., W.-L.T., G.R., A.A.B., M.O., H.G., K.M.S. and S.V.T. conducted the experiments. J.R.B., W.-L.T., G.R., A.A.B., K.M.S. and S.V.T. analysed the data. J.R.B., W.-L.T., K.M.S. and S.V.T. prepared the figures. W.-L.T., K.M.S. and S.V.T. wrote the manuscript.

\section{Additional information}

Supplementary Information accompanies this paper at http://www.nature.com/ naturecommunications

Competing financial interests: The authors declare no competing financial interests.

Reprints and permission information is available online at http://npg.nature.com/ reprintsandpermissions/

How to cite this article: Blount, J. R. et al. Ubiquitin-binding site 2 of ataxin-3 prevents its proteasomal degradation by interacting with Rad23. Nat. Commun. 5:4638 doi: $10.1038 /$ ncomms5638 (2014) 